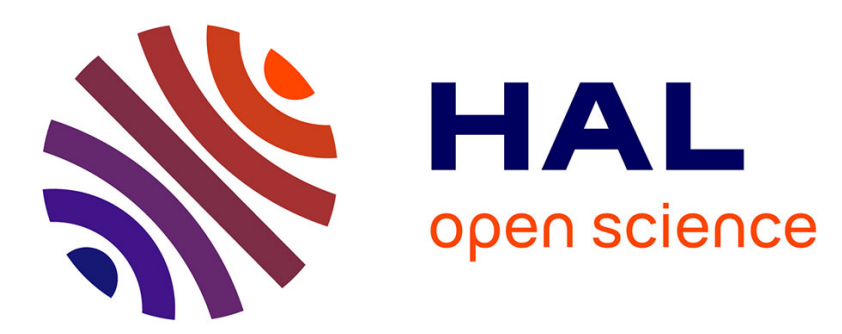

\title{
A novel procedure for coupling QoS control and handover decision for mobility management
}

Anis Zouari, Lucian Suciu, Jean-Marie Bonnin, Karine Guillouard

\section{To cite this version:}

Anis Zouari, Lucian Suciu, Jean-Marie Bonnin, Karine Guillouard. A novel procedure for coupling QoS control and handover decision for mobility management. ICWMC'08 : fourth international conference on wireless and mobile communications, Jul 2008, Athens, Greece. 10.1109/ICWMC.2008.28 . hal02901370

\section{HAL Id: hal-02901370 \\ https://hal.science/hal-02901370}

Submitted on 17 Jul 2020

HAL is a multi-disciplinary open access archive for the deposit and dissemination of scientific research documents, whether they are published or not. The documents may come from teaching and research institutions in France or abroad, or from public or private research centers.
L'archive ouverte pluridisciplinaire HAL, est destinée au dépôt et à la diffusion de documents scientifiques de niveau recherche, publiés ou non, émanant des établissements d'enseignement et de recherche français ou étrangers, des laboratoires publics ou privés. 


\title{
A Novel Procedure for Coupling QoS Control and Handover Decision for Mobility Management
}

\author{
Anis Zouari, Lucian Suciu, Jean Marie Bonnin, and Karine Guillouard
}

\begin{abstract}
Providing QoS for nomadic hosts remains a critical issue since new emerging wireless technologies may present different behaviours in terms of QoS, security or cost. Within this heterogeneous environment, especially if a handover occurs, keeping the same QoS level for one application may require some methods to negotiate the QoS provided in each network. In this paper, we propose to adopt a proactive and dynamic negotiation approach coupled with the handover decision algorithm implemented in [1]. This method is compared to two existing solutions ${ }^{1}$ developed in the Daidalos IST project [2] and in the Ambient Network IST project [7].
\end{abstract}

Index Terms-QoS negotiation, handover execution, handover decision algorithm.

\section{INTRODUCTION}

$\mathrm{T}$ HE rapid development of several wireless technologies (e.g. UMTS, WLAN, WiMAX, etc.) provides new possibilities in providing wireless network access to nomadic hosts. Thus, they are gaining popularity in various environments such as: enterprise, campus, home and personal access networks. However, user mobility remains a critical issue since new emerging applications may have strict QoS requirements in terms of transmission delay, throughput, loss rate, etc. Furthermore, this heterogeneous environment is marked by changes in topologies and availability of resources. For this reason, keeping the same QoS level of one application session is made more difficult each time a host switches to a new radio access technology. Thus, there is a great need to propose methods to negotiate provided QoS in each radio access network in order to improve the performance of handover decision mechanisms.

Among the proposed methods, stands the reactive approach adopted in the Daidalos IST project ([4] and [5]). In this case, the QoS negotiation is triggered after the execution of a probable handover. Based on received QoS information about candidate point of attachments (PoAs), mobility manager in Daidalos decides which is the most appropriate PoA for

${ }^{1}$ Daidalos and Ambient Network Ist projects aim to provide end-to-end QoS in heterogeneous environment including several mobile systems. They propose a global solution specifying network architecture with QoS and mobility management schemas. For this reason, in this paper, we have made the comparison of our proposal with the schemas coming out from these two projects. Notice that there is more standardized work done in this area. However, these proposals deal with QoS management independently from mobility management. handover and triggers the handover execution. If QoS in the new PoA is degraded, Daidalos enables the host to start QoS negotiation process, in order to request more resources. The disadvantage of the reactive method adopted in Daidalos is that a QoS negotiation cannot be initiated before the mobile node switches to its new PoA. For instance, if QoS negotiation established with new PoA fails, while it would succeed with another candidate PoA, that unsuitable PoA has been dropped from candidate list during interface selection phase.

To mitigate this problem, the Ambient Network (AN) IST project implements a proactive approach based on a dynamic negotiation of service level specifications (SLS) agreements ([8] and [9]). In fact, based on the peer to peer concept, each AN sub-domain advertises the QoS, which it can provide, to the other AN sub-domain. On receiving this advertisement, if an AN sub-domain is interested in establishing an SLS agreement, it may start QoS negotiation with the advertiser AN sub-domain. In AN, a new SLS can trigger a handover. In this case, they guarantee that the mobile node will switch to a new PoA that satisfies its QoS requirements. The disadvantage of this method is that limited resources in a network, such as power and bandwidth, will be used intensively because a QoS control message is advertised through the whole network periodically. Moreover, in this environment, the operator has great difficulty controlling its network resources.

If providing QoS is an important criterion of the performance of a QoS management method, network operators have to take into account several parameters while designing their networks such as: signalling overhead, functional properties and deployability. Thus, to reduce the QoS signalling overhead cost of the AN method, we propose a new method based on coupling QoS negotiation with the handover decision algorithm presented in [1]. This algorithm is based on distributing the handover decision function over multiple entities within networks and terminals. Therefore, this approach allows the construction of a handover decision chain by specifying which entities are able to control handover decision. Notice that a set of decision parameters is associated to each entity belonging to this chain. The initial results, given in [1], lead us to focus on integrating QoS negotiation during handover decision phase. In this context, each entity in the decision chain checks its local decision parameters. If some of these parameters are not satisfied, QoS management mechanism starts. At this point, a QoS negotiation chain, specifying the entities which will execute the QoS negotiation protocol, is constructed. In this case, negotiation entities exchange only the parameters to be negotiated. For this 
reason, an advantage of our method is the reduction of the QoS signalling overhead.

This paper is structured as follows. Section II and III presents an example of QoS negotiation scenario during the handover in the case of Daidalos and AN respectively. Then, section IV presents the scenario of QoS negotiation as it is implemented in our proposition. Section $V$ gives a comparative analysis of the afore-mentioned mobility scenarios. Finally, concluding remarks are given in section VI.

\section{DAIDALOS APPROACH}

\section{A. Overview}

One of the most challenging tasks of Daidalos project [2] is to provide end to end QoS for heterogeneous environments. For this reason, Daidalos implements DiffServ architecture to support QoS in the core network and to achieve scalability and performance. In addition, it introduces a hierarchical QoS architecture definition with new signalling schemes. This architecture is mainly composed of entities named QoS brokers (QoSBs). Two different types of QoSBs are defined: access network QoSB (ANQoSB) and core network QoS (CNQoSB). ANQoSB assures that QoS is provisioned in the access routers of the access network and participates in the handover process. In fact, it assures that resources are correctly reserved in a new end to end connection path. CNQoSB is the entity responsible of core network resource management. It is also a proxy element during the interdomain mobility management process.

\section{B. Handover execution diagram sequence}

In this, section we focus on the example of MN initiated handover (for more details about handover management in Daidalos see [3]). As depicted in Fig. 1, handover process can be divided into three phases: preparatory phase, handover execution phase and QoS control phase. In the preparatory phase (messages 1 and 2), MN receives QoS information from candidate access router (CaAR) about attached PoA. Based on this obtained information, the $\mathrm{MN}$ decides which the most appropriate PoA for handover is. It has to be noticed that the chosen PoA may not satisfy QoS requirements. However, it is the candidate PoA which provides the highest possible QoS.

At this point the handover execution phase starts (messages from 3 to 10). MN sends a Router Solicitation Proxy message (3) to its current access router (CuAR) with the chosen CaAR. The CaAR forwards the request for approval to candidate ANQoSB (Ca ANQoSB) to which CaAR is attached. Upon receiving this request, the $\mathrm{Ca} A N Q o S B$ verifies the availability of the required QoS, informs the CaAR (5) of the QoS requirements and sends back to the CaAR its handover decision (6). After this, the CuAR informs MN that it can now move to the new network (7). As soon as MN receives the Proxy Router Advertisement message, it switches to the new network $(8,9$ and 10$)$.

In case of service degrading, MN starts QoS control phase (messages from 11 to 18). In this phase MN takes the initiative of contacting the Ca ANQoSB via its CaAR in order to ask it to reserve more resources (11 and 12). Upon receiving the QoS flow request, $\mathrm{Ca}$ ANQoSB sends a request to its CNQoSB to perform core reconfiguration. Based on

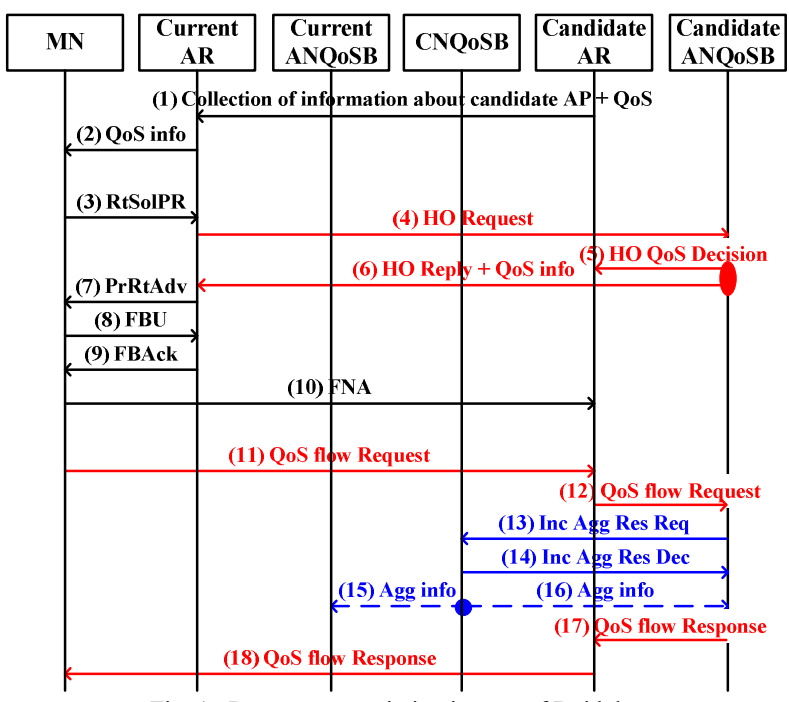

Fig. 1. Resource negotiation in case of Daidalos

measurements, CNQoSB reconfigures the bandwidth reserved for the aggregates and informs all attached ANQoSBs of the resources availability (or unavailability) in the core for particular classes and on the paths between particular subdomains (14, 15 and 16). At the end, Ca ANQoSB sends its decision about the received $\mathrm{MN}$ request (17 and 18).

\section{AMBIENT NETWORK APPROACH}

\section{A. Overview}

Unlike DAIDALOS, AN IST project [7] proposes a separation of the control plane from the data transport plane. Thus, AN has defined common control space responsible for creating an edge-to-edge networking environment. This control plane has been subdivided into several functional areas (e.g. context provisioning, mobility, connectivity controller, QoS, etc.) in order to manage the establishment of different connections. To hide the diversity of radio access technologies to the control plane, an ambient connectivity plane has been defined. It has to be noted that each entity in an ambient network regroups the control and the connectivity functions in two different planes. In addition, an AN QoS entity (AQE) has been specified to control QoS in each AN sub-domain. This entity is responsible of SLS negotiation.

\section{B. Handover execution diagram sequence}

As specified in [9], the AN adopts a peer to peer approach to negotiate QoS for the path to be established between two entities during handover. The core ambient network (CAN) advertises its services through SLSs to their neighbour radio access networks. Each SLS includes the QoS supported in CAN to reach a specified radio access network.

As depicted in Fig. 2, the handover process in AN can be divided into two phases: QoS control phase and handover execution phase (for more details about handover management in AN see [10] and [11]). In this section, we focus only on the QoS control phase via a scenario example of $\mathrm{MN}$ which moves from an $\mathrm{AN}$ sub-domain managed by an AN QoS 


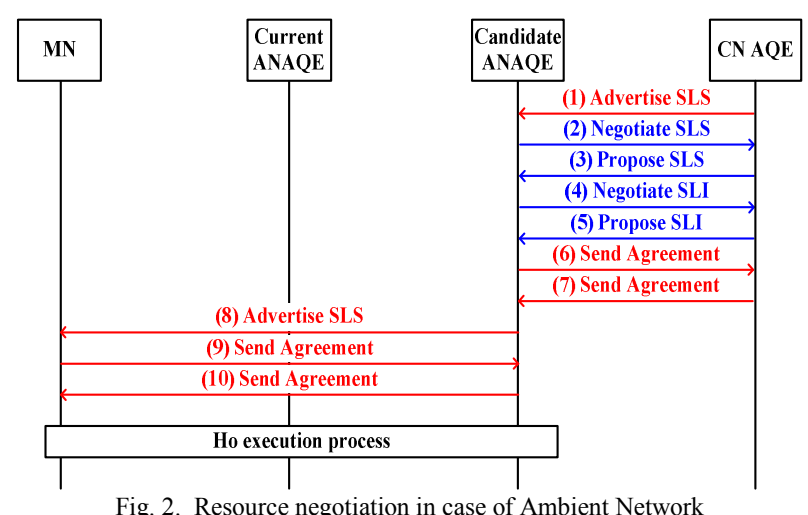

entity (AQE), named current ANAQE, to a candidate AN subdomain managed by a candidate ANAQE. In the QoS control phase, we first negotiate an SLS between CNAQE and candidate ANAQE, afterward we negotiate an SLS between candidate ANAQE and MN. SLS negotiation is basically an exchange of three messages (e.g. messages 1,6 and 7). For example, in the negotiation of SLS between the access network and MN, ANAQE first send an advertise SLS message (8) to MN containing information about available QoS. On receiving this message and if the received SLS satisfies the MN QoS requirements, $M N$ accepts the establishment of SLS and sends a send agreement message to the ANAQE to inform it. In order to finalise the SLS establishment process, the ANAQE sends back a send agreement message. At this point the handover execution phase starts. In this phase MN chooses the mobility protocol to execute and merges to the new radio AN sub-domain.

If the received SLS does not satisfy local QoS requirements (the case of candidate ANAQE), they are able to negotiate this SLS and propose new SLS (messages 2, 3, 4 and 5).

\section{HIERARCHICAL AND DYNAMIC APPROACH}

\section{A. Overview}

As presented in [1], the distribution of handover decision algorithm can provide greater flexibility to network operators as they will be able to achieve fine-grained handover decisions. In this approach, if the QoS requirements are not satisfied in a candidate connection path, the probability that $\mathrm{MN}$ switches to this candidate path is decreased. In some cases, QoS negotiation may address this problem and so the probability that MN will be "the best connected" can increase. Thus, the performance of the distributed decision algorithm is improved by adding some QoS management mechanisms in order to include QoS constraints in the decision motivations.

The distributed decision algorithm in [1] is based on utility function model implemented in each QoS manager. When this algorithm is started, each QoS manager first negotiates its associated list of parameters. Then, it takes a partial decision (i.e. manager decides to admit or to refuse application traffic according to its allocated parameters). Finally, it sends its partial decision to lower node (e.g. another manager or terminal). Our proposition is to start QoS negotiation as soon

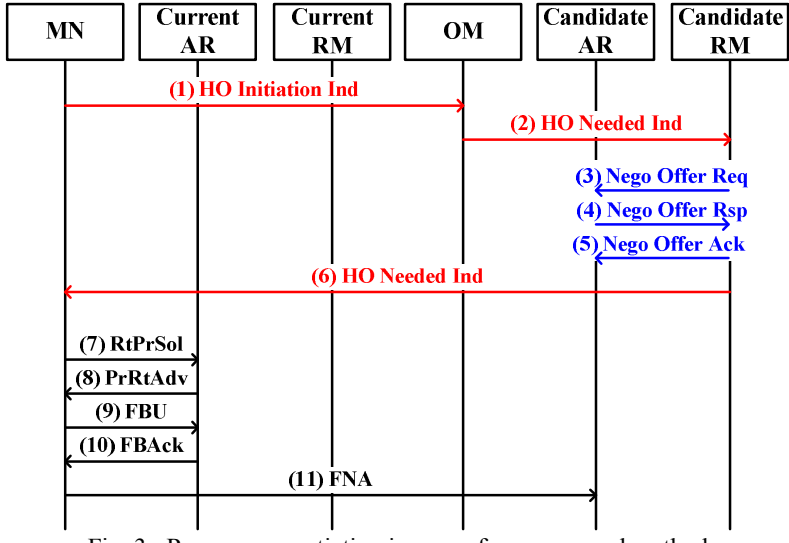

Fig. 3. Resource negotiation in case of our proposed method

as the manager decides to refuse the admission because QoS requirements are not satisfied.

In our approach, we have adopted generic protocol of negotiation which gives to two negotiation entities the ability to communicate together. This protocol can be mapped to several current negotiation protocols. In addition, this protocol has defined generic object to transport QoS parameter required values and their current values provided by the network. During negotiation process, three messages are needed: QoS negotiation request message, QoS negotiation reply message and QoS negotiation acknowledgement message. First two messages are used to exchange any proposed QoS parameters. Third message is used to acknowledge the new mutually established agreement.

\section{B. Handover execution diagram sequence}

As in AN, our proposed handover process can be divided into two phases: QoS control coupled with handover decision phase and handover execution phase (for more details see Fig. $3)$. Both phases are set out in this section via a mobility scenario example where the handover decision is distributed between three entities: $\mathrm{MN}$, radio QoS manager (RM) and operator QoS manager (OM). In the first phase, MN first discovers the available candidate RAs. Then, it sends a handover initiation indication message (1) to its OM with the list of selected candidate ARs. Upon receiving this message, $\mathrm{OM}$ verifies if the QoS parameters stored locally satisfies the QoS requirements. If they are satisfied, OM computes its partial decision score and sends handover needed indication message (2) to each RM having an attached AR from the received selected list. Here we assume that there is only one candidate AR. On checking the local QoS parameters, the candidate RM discovers that QoS is degraded. For this reason, it starts a QoS negotiation process to request more resources. This is followed by the exchange of two messages ( 3 and 4) between the candidate RM and its attached AR. In the first message, the candidate RM requests a new QoS offer. On receiving this message and based on current measurements, the candidate AR proposes a new offer and forwards it to the candidate RM. This latter parses the new offer and decides if QoS requirements are now satisfied by the new offer. In this case, the candidate RM sends an acknowledgement message 
(5), completes the computation of partial score with the negotiated local QoS parameters and sends a handover needed indication message (6) to MN. Similarly to the RM, on receiving the higher level partial score, $\mathrm{MN}$ checks its local QoS parameters, computes the final scores and then takes the final handover decision (i.e. it chooses a point of attachment to be connected to).

At this point the handover execution phase starts (message from 7 to 11). In this scenario, as for Daidalos scenario, the fast handover mobile IP protocol [12] is chosen as mobility management protocol.

\section{PERFORMANCE EVALUATING AND COMPARING ANALYSIS FOR A MOBILITY SCENARIO}

This section is mainly interested in analyzing the QoS negotiation process. This negotiation process is started when there are some QoS parameters in the new connection path which need to be negotiated. In the following, we first make a functional comparative analysis of the aforementioned methods. Afterwards we give a conceptual analysis of QoS negotiation process performance.

\section{A. Functional analysis}

As illustrated in previous sections, each QoS control method adopts different designs. Daidalos has chosen the QoSB to be the only entity in the network responsible for handling received QoS requests. In Daidalos, each ANQoSB is also able to start QoS negotiation process with its correspondent CNQoSB in order to request more resource reservation. However, because QoSB are usually located deeply within networks, these approaches are not fast enough to follow the rapid changes of radio channels capabilities.

Unlike Daidalos, AN implements a model based on bilateral agreements in order to support end-to-end QoS. In this approach, each AN advertises to its neighbours a set of possible QoS agreements, via which the advertiser AN promises a certain level of QoS to a certain amount of traffic. If one neighbour $\mathrm{AN}$ is interested by the advertised resources to handle a QoS request, it establishes a QoS agreement with the advertiser. Such a solution allows the QoS negotiation of SLS without taking into account different preferences on the end-terminal side, and without considering the constraints we may encounter from the network side (i.e., operator policies). This means that some negotiated SLS may not be used which induces supplementary signalling load in the networks.

To mitigate the problem of loaded network, our proposed method suggests the distribution of the QoS control between several entities in the network and end-terminals. In fact, QoS requirements are checked during the decision algorithm and the Qos negotiation may be locally triggered if needed. This reduces the amount of exchanged QoS signalling. In addition, as QoS parameters are collected from several entities located in different hierarchical levels (e.g. signal to noise ratio is measured by end terminal, connection cost is measured in core network, etc.), the distribution of QoS control improves the performance of handover process. In the following section, all these points are studied in more details.
TABLE I

QOS NEGOTIATION SIGNALLING MEASUREMENTS FOR EACH METHOD

\begin{tabular}{llll}
\hline \hline & AN method & $\begin{array}{l}\text { Daidalos } \\
\text { method }\end{array}$ & $\begin{array}{c}\text { Proposed } \\
\text { method }\end{array}$ \\
\hline $\begin{array}{l}\text { QoS control messages } \\
\text { QoS negotiation }\end{array}$ & 0 & 7 & 3 \\
messages & 10 & 4 & 3 \\
Signalling overhead & $10 *(\mathrm{O}+$ & $11 * \mathrm{O}+6$ & $6 * \mathrm{O}+2 * \mathrm{~S}_{\mathrm{D}}$ \\
& $\left.\mathrm{S}_{\mathrm{SLS}}\right)$ & $* \mathrm{~S}_{\mathrm{SLS}}$ & $+2 * \mathrm{~S}_{\mathrm{T}}$ \\
\hline \hline
\end{tabular}

\section{B. Study assumptions}

In this QoS negotiation study, we assume:

- The candidate path can not provide enough resources. Transmission delay and throughput should be negotiated. However, after the negotiation process the candidate path is able to receive $\mathrm{MN}$ flows.

- In the case of AN scenario, both parameters are not satisfied in the SLS negotiated between core network and radio access network. In the case of our proposed scenario, both parameters are associated to the RM.

- The messages exchanged during QoS negotiation uses the User Datagram Protocol (UDP) [13] at transport layer. Consequently, these messages must be contained in one UDP/IP packet. Each message is mainly composed of two parts: protocol headers (also named overhead) and data. As previously stated, QoS signalling message travels over an UDP segment which is encapsulated in an IP packet. For this reason, the total overhead, namely $\mathrm{O}$, needed to send a QoS signalling message is the sum of the overhead introduced by UDP and IP protocols. The second part of QoS signalling message contains SLS QoS information.

- In the following sections, $\mathrm{S}_{\mathrm{SLS}}$ refers to the size of SLS QoS data in a message. $S_{D}$ is the size of transmission delay information in message. $\mathrm{S}_{\mathrm{T}}$ is the size of throughput information in message. Notice that $\mathrm{S}_{\mathrm{SLS}}$ is less than the sum of $\mathrm{S}_{\mathrm{D}}$ and $\mathrm{S}_{\mathrm{T}}$. for numerical results, we assume that $\mathrm{S}_{\mathrm{SLS}}=4 *$ $\mathrm{S}_{\mathrm{D}}, \mathrm{S}_{\mathrm{SLS}}=4 * \mathrm{~S}_{\mathrm{T}}$ and $\mathrm{O}=56$ bytes.

\section{Conceptual analysis}

In this paper we have analysed the behaviour of our method, by comparing it to the methods implemented in Ambient Network and Daidalos IST projects. Thus, we measured the signalling overhead and the number of needed messages for QoS managements. Notice that during QoS management two types of messages are exchanged: QoS control messages and QoS negotiation messages. QoS control messages are used for checking if QoS requirements are satisfied. QoS negotiation messages are used to negotiate new parameters values. The results are summarised in Table I.

Fig. 4, shows that in our method we have the lowest number of exchanged QoS messages. This represents the first step of reducing network load. This is also illustrated in Fig. 5 which gives the total amount of QoS data for all studied methods. We notice that our proposed method induces lower signalling overhead. Moreover, it showed a better performance in what concerns scalability with the number of established negotiation sessions. However, our methods may have 


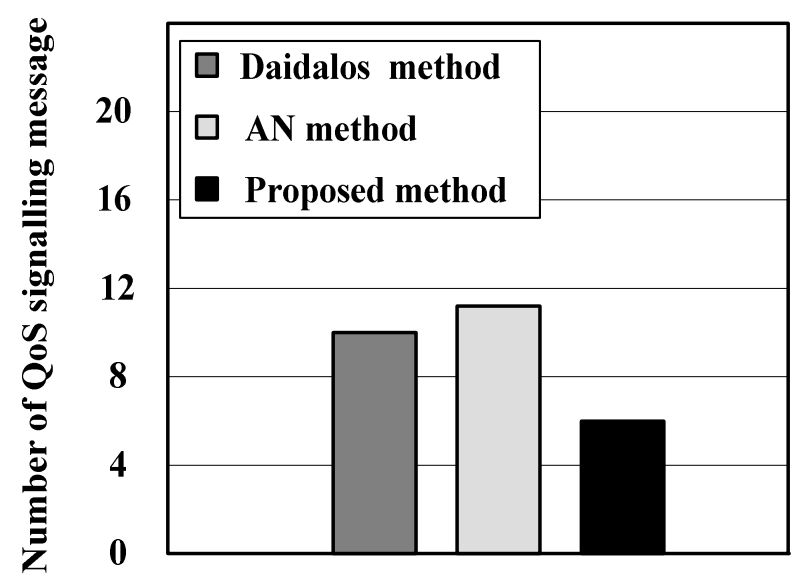

Fig. 4. Number of QoS signaling message during Handover for the three studied methods

scalability problems with increased number of available applications. In fact, in our approach a negotiation is established for each user's application. On the other hand, Ambient Network negotiates an SLS to be used independently of the applications. This makes it difficult for operator to control its network resources.

\section{CONCLUSION}

This paper proposes a new method for negotiating QoS parameters coupled with the decision algorithm. This approach aims at developing an innovative vision of the recent research done on proactive negotiation methods. Likewise, the introduced optimization makes use of the fact that the QoS parameters are collected from several entities located in different hierarchical levels. Accordingly, this information can be used locally during QoS negotiation and thereby we can reduce the amount of QoS data included in QoS signalling messages. An evaluation of distributing QoS parameters over multiple entities able to communicate together has been also performed in this paper. Thus, we compare the behaviour of our method to the methods adopted in Ambient Network and Daidalos in what concerns the number of messages needed to negotiate QoS parameters and the overhead generated by the QoS signalling messages. Our initial analysis points out an improvement in the QoS management if our proposed method is adopted. We are currently assessing the performance of our approach by implementing it in OPNET.

\section{REFERENCES}

[1] L. Suciu and K. Guillouard. "A hierarchical and distributed handover management approach for heterogeneous networking environments". The $3^{\text {rd }}$ International Conference on Networking and Services, Athens, Greece, June 2007.

[2] Amardeo Sarma, "Daidalos Five Key Concepts", Euro-India Workshop on Next Generation Wireless Technologies, Calcutta, India, November 2006.

[3] Rui L. Aguilar, Susanna Sargento et al, "Scalable QoS aware Mobility for future Mobile operators", IEEE communication Magazine, June 2006.

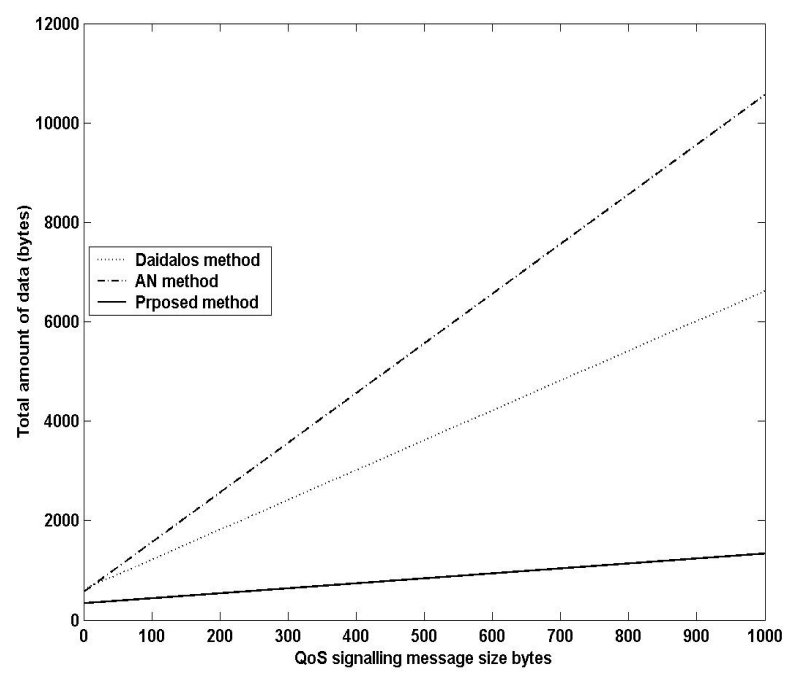

Fig. 5. QoS signaling overhead cost during mobility scenario

[4] Nikos Koutsouris et al, "A Performance Management architecture for QoS enabled heterogeneous networks", IST Mobile Summit, Budapest, Hungary, July 2007.

[5] G. Carneiro and M.Ricardo, "QoS Abstraction Layer in 4G Access Networks", Telecommunications Systems, Springer, August 2007.

[6] P. PACYNA, J. GOZDECKI, K. ŁOZIAK and A. JAJSZCZYK, "Mobility Across Multiple Technologies — the Daidalos Approach ", Special Issue on Next Generation Network Technologies, Interdisciplinary Information Sciences, Octoer 2006.

[7] A. Schieder, P. Schoo, J.Gebert, D. Zhou, K. Balos and M. Kano, "The Reference Points of an Ambient Network", IST Mobile Summit, Budapest, Hungary, July 2007.

[8] I. Psaras, L. Mamatas and P. Mendes, " INQA: InterNetwork QoS Agreements - A New Protocol for Dynamic SLS Control in Next Generation Networks", Workshop on Networking in Public Transport, Ontario, Canada, Auguste 2006.

[9] J. Andrés-Colás, C. Pinho, P. Mendes, Y. Wang, and J. Ruela, "Internetwork Quality of Service Agreements among Ambient Networks", Towards the QoS Internet Workshop, Coimbra, Portugal, May 2006.

[10] A. Surtees, R. Aguero, J. Eisl and M. Georgiades, "Mobility Management in Ambient Networks", the IEEE 65th Vehicular Technology Conference, Dublin, Ireland, April 2007.

[11] IST-Ambient Networks, "Mobility Architecture and Concepts Annex", Internal Deliverable D4.2, March 2005.

[12] R. Koodli (ed.), "Fast Handovers for Mobile Ipv6", IETF RFC 4068, July 2005.

[13] J. Postel, "User Data Protocol", IETF RFC 768, August 1980. 